\title{
The Surrounding Rock Pressure Research of Tunnel Group with Large Section and Three-hole of Small Neighborhood
}

\author{
Yue-Guang $\mathrm{HE}^{1, \mathrm{a},{ }^{,} \text {, Lei-Sen SHAO }}{ }^{1, \mathrm{~b}}$, Meng ZHOU ${ }^{1, \mathrm{c}}$ \\ ${ }^{1}$ School of Transportation Engineering, Changsha University of Science and Technology, Changsha \\ 410004, China \\ ahyg@csust.edu.cn, b544012001@qq.com, c1121874907@qq.com \\ ${ }^{*}$ Corresponding author
}

Keywords: Tunnel, Surrounding Rock Pressure, Depth, Earth Covering Thickness.

\begin{abstract}
Derivation of parallel three-hole of small spacing Tunnel in shallow non- bias case, surrounding rock pressure formula in sequence of first side hole after middle hole excavation, drawn the corresponding computational model For the study of parallel three holes of small clear space tunnel surrounding rock pressure in the case of size and distribution in a shallow non- bias, and impact of the law by earth covering thickness change, conducted a comparative calculation When the tunnel clear distance is certain, on the whole, three holes tunnel outside vertical pressure and lateral pressure with depth increases increasing, and approximately linear growth By contrast, drawn parallel to the three-hole and small spacing tunnel excavation, three holes to force state has deteriorated compared with norms calculated in single hole excavation, the calculation specification requires only symmetric stress state is chosen not safe conclusion

The most representative works of our country tunneling engineering tunnel surrounding rock pressure calculation is rock pressure single hole tunneling calculations The actual engineering practice, parallel three holes and large section of small clear space tunnel gradually use, its "multi-cavern effect" obviously, but no suitable surrounding rock pressure calculation In addition, cavern excavation sequence between the value of the load great influence, If confined cavern final state, obvious un-reasonable To this end, the theoretical calculation method to explore parallel three holes and large section of small clear space tunnel.
\end{abstract}

\section{Load Calculation Mode}

For convenience of calculation to simplify calculation model basic hypothesis and simplified as follows $[1,2]$

(1) Calculation analysis process, it is assumed as a single continuous medium surrounding rock; horizontal ground and are shallow tunnel; between three holes equally spaced and horizontal direction parallel arrangement According to above work wan can take the middle hole medial axis for the sector, taking half for analysis

(2) Consider the interaction of adjacent tunnel construction, construction order is sided holes(left and right hole) excavation first(Simultaneously or successively excavation), then middle hole excavation When the excavation sided holes, without considering the interaction between the two holes, Within two lateral tunnel surrounding rock pressure calculation formula is consistent with the specification in the case of a single hole in the tunnel; when excavation middle hole, Surrounding the outer edge of the sided hole pressure remains unchanged, On the interaction region (medialand vault) the force correction

(3) Due to the small neighborhood tunnel clear distance D is generally small, Calculate the left hole and the middle hole rock pile within fracture plane intersection point to two side hole distance $\mathrm{D}_{1}, \mathrm{D}_{2}, \mathrm{D}_{1}$ can be $\mathrm{D} / 2$ approximately 


\section{Load Calculation}

\section{Load Size and Distribution}

Before Middle Hole Excavation Load Calculation. When left hole and right hole excavation first, there is no impact on each other, so it is the same with a single tunnel excavation, surrounding rock pressure in medial and lateral of the tunnel is symmetric distribution, its surrounding rock pressure distribution pattern shown in Figure 1
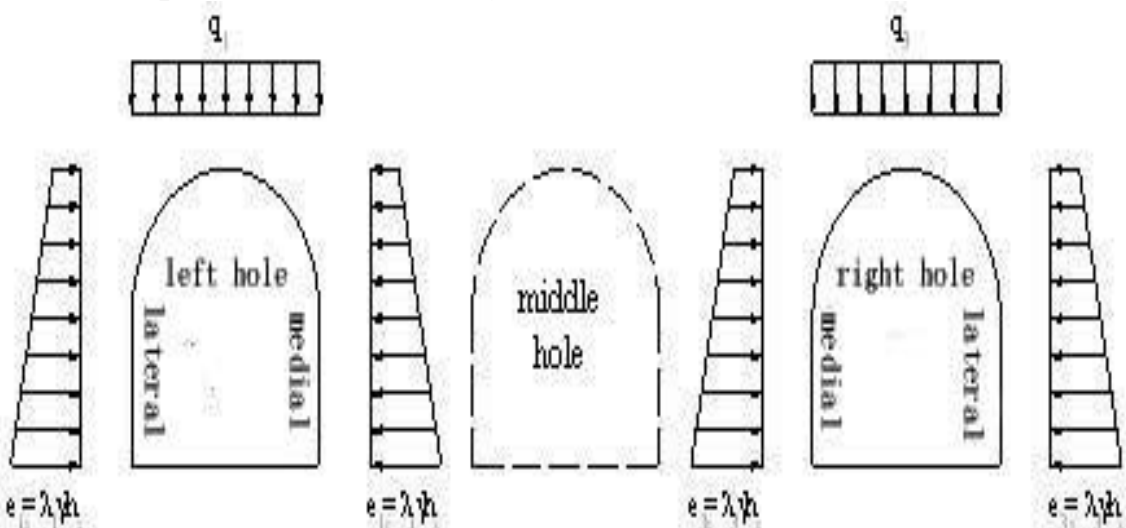

Fig. 1 The Left and Right Tunnel Excavation, Surrounding Rock Pressure Distribution Patterns in Around Hole Tunnel Shallow Non- bias

Load calculation formula is as follows [3]:

sided holes lateral horizontal pressure: $e_{i}=\lambda \gamma h_{i}\left(\mathrm{kN} / \mathrm{m}^{2}\right)$

vault vertical pressure: $\quad q=\gamma \mathrm{H}\left[1-\frac{\mathrm{H}}{B} \lambda \tan \theta\right]_{(\mathrm{kN} / \mathrm{m} 2)}$

which lateral pressure coefficient:

$\lambda=\frac{\tan \beta-\tan \varphi_{c}}{\tan \beta\left[1+\tan \beta\left(\tan \varphi_{c}-\tan \theta\right)+\tan \varphi_{c} \tan \theta\right]}$

Fracture angle $\beta$ are separately $\beta_{1}, \beta_{2}$ as left hole, right hole Produce maximum thrust by $T_{1}, T_{3}:$

$$
\tan \beta_{1}=\tan \varphi_{c}+\sqrt{\frac{\left(\tan \varphi_{c}^{2}+1\right) \tan \varphi_{c}}{\tan \varphi_{c}-\tan \theta}}
$$

in which, $h_{i}$ is calculated point depth, that calculation points to the distance from the ground $(\mathrm{m})$ 。

After Middle Hole Excavation Load Calculation. After middle hole excavation, two lateral tunnel surrounding rock pressure remained unchanged, the rupture slip body formed in the inside surrounding rock were the original triangle landslide body becomes a trapezoid landslide body Fracture plane formed on both sides of the middle hole tunnel in the lower part of the horizontal angle $\beta_{2}$

Based on the hypothesis (1), the distribution of sided holes will be left holes surrounding rock loads for example

(1) Middle hole lateral horizontal pressure 


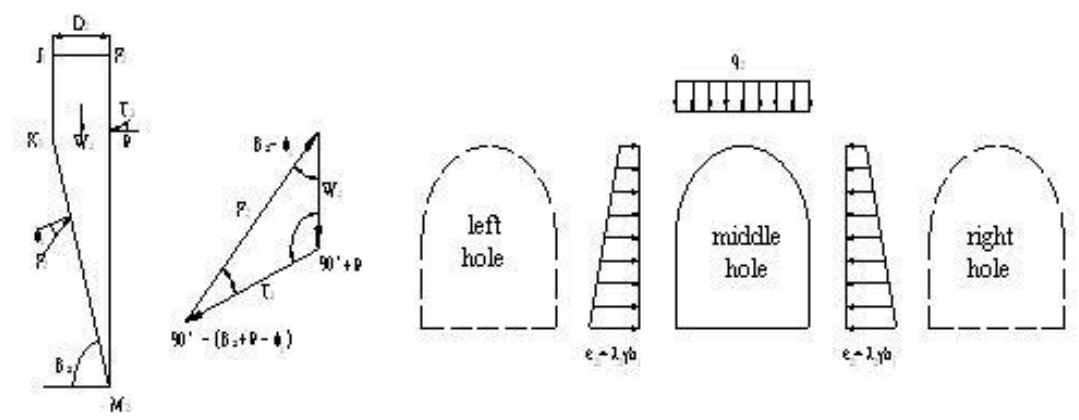

Fig. 2 Middle Hole Tunnel Excavation, Hole Tunnel Surrounding Rock Side Pressure Diagram with the Surrounding Rock Pressure Distribution Patterns

According to the calculation of sine theorem and force equilibrium conditions can be obtained in frictional rock surrounding the middle hole on both sides of the body resistance $T_{2}$ is as follows:

$$
\begin{gathered}
W_{2}=\frac{D_{2}\left(h+h-D_{2} \tan \beta_{2}\right)}{2} \gamma=\frac{D_{2}\left(2 h-D_{2} \tan \beta_{2}\right)}{2} \gamma=\frac{D\left(4 h-D \tan \beta_{2}\right)}{8} \gamma \\
T_{2}=\frac{\sin \left(\beta_{2}-\varphi_{c}\right)}{\sin \left[90^{\circ}-\left(\beta_{2}-\varphi_{c}+\theta\right)\right]} W_{2}=\frac{D \gamma\left(4 h-D \tan \beta_{2}\right) \sin \left(\beta_{2}-\varphi_{c}\right)}{8 \sin \left[90^{\circ}-\left(\beta_{2}-\varphi_{c}+\theta\right)\right]}
\end{gathered}
$$

Set $\lambda_{2}$ is lateral pressure coefficient of the middle hole, according to the definition of lateral pressure coefficient Shows:

$$
T_{2}=\frac{1}{2} \gamma h^{2} \frac{\lambda_{2}}{\cos \theta}
$$

Joint Formula (5), (6), (7), we get:

$$
\lambda_{2}=\frac{\left(\frac{D}{h}-\frac{D^{2}}{4 h^{2}} \tan \beta_{2}\right)\left(\tan \beta_{2}-\tan \varphi_{c}\right)}{1+\tan \beta_{2}\left(\tan \varphi_{c}-\tan \theta\right)+\tan \varphi_{c} \tan \theta}
$$

When generating maximum thrust $T_{2}$, get $\frac{d \lambda_{2}}{d\left(\tan \beta_{2}\right)}=0$

Fracture angle $\beta_{2}$ calculated to produce maximum thrust $T_{2}$ when available as follows:

$$
\tan \beta_{2}=-\frac{1}{\tan \left(\varphi_{c}-\theta\right)}+\sqrt{\frac{\tan \varphi_{c}^{2}+1}{\tan \varphi_{c}-\tan \theta}\left[\frac{1}{\tan \left(\varphi_{c}-\theta\right)}+\frac{4 h}{D}\right]}
$$

The inside of the left hole to get the medial lateral pressure if values $\lambda_{2}$ into equation (1)

(2) The middle hole vault vertical pressure

Gravity of Overburden Rocks on the middle hole tunnel vault of is $\mathrm{W}_{\text {middle }}$, both sides are being slip surface resistance as $T_{2}$, possible to obtain the total vertical pressure acting on the plane is:

$$
\mathrm{Q}_{\text {2shallow }}=\mathrm{W}_{\text {middle }}-2 T_{2} \sin \theta=\gamma H\left[\mathrm{~B}-\mathrm{H} \lambda_{2} \tan \theta\right]
$$

After conversion into a uniform load vault vertical pressure for the middle hole:

$$
q_{2}=\frac{Q_{2 \text { shallow }}}{\mathrm{B}}=\gamma H\left\lfloor 1-\frac{H}{B} \lambda_{2} \tan \theta\right\rfloor
$$


The inside edge of the sided hole surrounding rock pressure calculation can be calculated with reference to the process of middle hole lateral horizontal pressure, Simplify the calculation load chart, establishment inside edge of the sided hole surrounding rock pressure distribution pattern, using the weight $W_{1}^{\prime}$ of surrounding rock and $T_{1}^{\prime}$ as soil on both sides of the tunnel vault crown settlement soil column imposed slip surface resistance, Calculate medial lateral pressure coefficient of the left hole as $\lambda_{1}^{\prime}$, set $\lambda_{1}^{\prime}$ into equation (1), then can get inside of the left hole to get the medial lateral pressure; Calculate vault vertical pressure of the left hole is equated with vault vertical pressure of the middle hole, using gravity of Overburden Rocks on the left hole tunnel vault is $\mathrm{W}_{\text {left }}$, both sides are being slip surface resistance is $T_{1}$ and $T_{1}^{\prime}$,we can get the total vertical pressure acting on the plane after the middle hole excavation, After conversion into a distributed loadvault vertical pressure for the left hole: and the paper assumes a linear variation between inside and outside the tunnel, surrounding Rock pressure calculation method and processes of right hole is similar with left hole[4,5,6].

\section{Tunnel Surrounding Rock Pressure Group Characteristics}

Select a different tunnel design parameters calculated by the above theories surrounding rock pressure, comparative analysis parallel three holes of small clear space tunnel surrounding rock pressure in the case of size and distribution in a shallow non- bias, and impact of the influence law by earth covering thickness change[7,8,9,10,11]

\section{Depth on the Rock Pressure}

Select the calculation parameters: a parallel three holes and Large section of small clear space tunnel, single tunnel double, excavation span $\mathrm{B}=14 \mathrm{~m}, H_{g}=12 \mathrm{~m}$, the type of surrounding rock is $\mathrm{V}$, severe of surrounding rock is $\gamma=20 \mathrm{kN} / \mathrm{m}^{3}$, internal friction angle $\varphi=24^{\circ}$, calculation friction angle $\phi_{\mathrm{c}}=45^{\circ}, \theta=27^{\circ}$, Tunnel clear distance $\mathrm{D}$ and depth $\mathrm{H}$ selected depending on the calculation conditions.

Equivalent load height is available [3]:

$$
h_{q}=0.45 \times 2^{5-1}[1+0.1 \times(14-5)]=13.7 m
$$

Figures 3 and 4 for parallel three-hole of small spacing Tunnel clear distance $\mathrm{D}=8 \mathrm{~m}$ of $\mathrm{V}$ surrounding rock, Three holes tunnel medial and lateral vertical pressure and lateral pressure and their ratio with depth change curve.

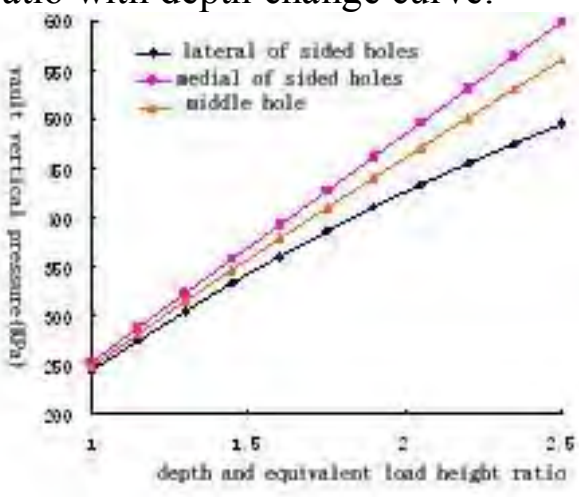

(a) Vertical pressure

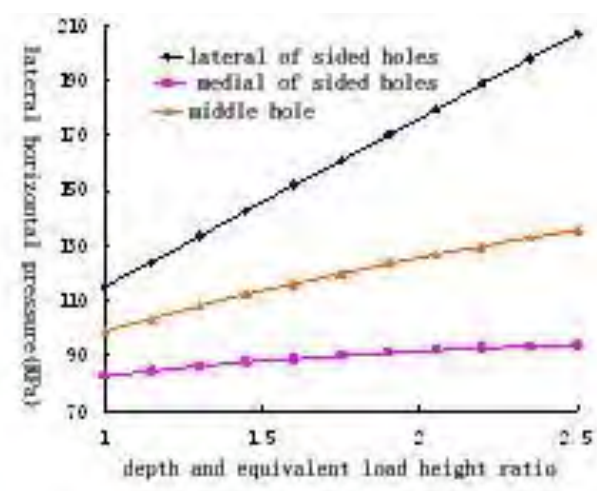

(b) Lateral pressure

Fig. 3 Surrounding Rock Pressure Curve with the Tunnel Depth of Parallel Three-hole of Small Neighborhood Tunnel Shallow Non - bias 


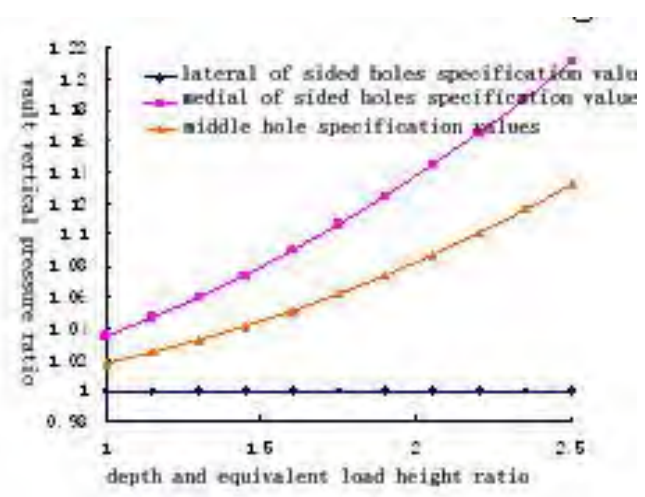

(a) Vertical pressure ratio

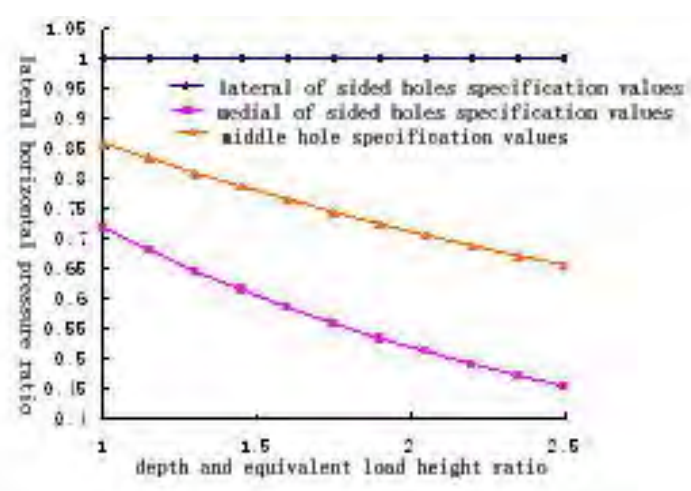

(b) Lateral pressure ratio

Fig. 4 Surrounding Rock Pressure Ratio Curve with the Tunnel Depth of Parallel Three-hole of Small Neighborhood Tunnel Shallow Non - bias

Figure 3 shows: on the whole, three holes tunnel outside vertical pressure and lateral pressure with depth increases increasing, and approximately linear growth First excavated left hole and right hole, their medial vertical pressure were greater than the lateral of tunnel, however, medial lateral pressure were less than lateral of tunnel, and with the tunnel depth increases, increasing the pressure difference between inside and outside is relatively large, show more obvious bias of the tunnel; surrounding rock pressure in the excavation of the middle hole then symmetrical distribution.

As apparent from Figure 4: compared with the standardized single-hole case, makes the first excavation of right and left hole Vertical pressure of medial of the vault are all greater than specification because middle hole excavation, and with increasing depth ratio is further increased, Vertical pressure of lateral of the vault are equal with specification; right and left hole which lateral pressure of medial are less than specification, their lateral pressure of lateral are equal with specification; the last excavation of middle hole lateral pressure symmetrical distribution, but both sides of lateral pressure than specification is small, which Vertical pressure of lateral of the vault is equal with specification; after excavation of middle hole, Making the first excavation of left and right hole have maximum vertical pressure of medial, medial the lateral vertical pressure of middle hole in the second, lateral of left and right hole is the smallest.

\section{Conclusion}

Through parallel three-hole of small neighborhood Tunnel,in a non-bias case shallow and excavation sequence of first sided holes then middle hole, derivation surrounding rock pressure calculation formula, a more reasonable calculation model and using the calculation model feature analysis, concluded that:(1) Under the order of excavation sequence of first sided holes then middle hole, surrounding Rock pressure of the side hole from the symmetric distribution becomes asymmetric distribution, Biased obvious effect on both sided holes, surrounding rock pressure of middle hole is symmetric distribution, stress characteristics is similar with structure of compressed tunnel, Three-hole stress state has deteriorated compared with code calculating when calculating a single tunnel excavation(2) Three holes tunnel surrounding rock pressure calculations by theoretical calculation results are different with code calculating, Conducting design calculations of supporting structure under parallel three-hole of small neighborhood Tunnel in shallow non- bias case, should calculate Separately for the left and right hole and the middle hole, not always the case, mere "feel" and experience a certain percentage of the load to amplification The specification only symmetrical stress state calculate is chosen not safe

\section{References}

[1]ZENG Jian The application of Neighborhood Tunnel in the mountain high-grade highways[J], East China Highway, 2010(5):82-84. 
[2]YANG JP, CHEN WZ, GUO XH Effect of supporting time on stability of small spacing roadway tunnel[J], Rock and Soil Mechanics, 2008(02): 483-490.

[3]The Design Specifications of the Railway Tunnel TB10003-2005 [M], China Railway Press.

[4]FENG MW Neighborhood Tunnel features, construction methods and points to note[J], China New Technologies and Products, 2010(09):41-42.

[5]LI DL,HU JY,HE QY,DIAO JZ Numerical Simulation of small spacing under different clear distance highway tunnel[J] West-China Exploration Engineering, 2007(06):134-137.

[6]ZHANG LY The small distance double tunnel excavation construction method[J] Shanxi Architecture, 2010(23):311-312.

[7]REN XH,WANG SH,WANG MQ,LIU Li Engineering geological conditions and diseases of Longyou large ancient underground caverns[J] Hunan University of Science and Technology University (Natural Science Edition), 2004(03):39-42.

[8]ZHOU ZL,LIU CW,ZHANG ZG Effect of Depth on Stress of Tunnel Surrounding Rock and Lining [J] Copper Engineering, 2010(03):1-4.

[9]WANG YC,SHANG YQ,XU XH Orthogonal back-analysis of geotechnical parameters and working state evaluation of lining of shallow buried tunnel[J] Journal of Central South University(Science and Technology), 2011(06):1764-1771).

[10]LIU FS, WEN JZ, WANG Cheng Analytical Study on Surrounding Rock Pressure of Tunnels by Back-Analysis on Periphery Displacements[J] Chinese Journal of Underground Space and Engineering, 2007(S1):1203-1207).

[11]LI XJ,XIONG Zheng,WANG MeiThe support research and countermeasures of Hydraulic Tunnel unstable surrounding rock excavation J] China Water Transport, 2008(10):157-158). 